\title{
Repository System Development using Partial Differential Equations
}

\author{
Prof. Muhamad Angriawan \\ Department of Computer Engineering, \\ IRC Russia
}

\author{
Article Info \\ Page Number: $118-121$ \\ Publication Issue:
}

Vol 71 No. 1 (2022)

\begin{abstract}
To visualize breast cancer, the procedure calls for expensive calculations, as well as a huge memory. In the majority of the previous investigations that have focused on breast cancer growth, they have reported the need for parallel computer systems development's distributed processors, as well as the distributed memory. Therefore, when parallel repository systems are developed based on important computation platforms, there tends to be benefits such as decreased costs and increased speed. In this study, it was established that the proposed simulation software exhibits several strengths. They included being friendly, multidimensional breast cancer visualization, and high performance estimation. Also, the software, in the wake of big data dominance in the healthcare sector, offers strength and real-time solution. The implication is that the proposed model would offer promising results regarding computer-aided decision-making relative to the screening for breast cancer, as well as disease treatment, clinical assessments, and diagnosis. Hence, parallel algorithm could be used as an ideal model of solving sequential algorithm's drawbacks, especially regarding time consumption.
\end{abstract}

Keywords: - Repository System, Partial Differential Equations etc.

\section{Introduction}

Recent statistics suggest that among women, the second deadliest form of cancer is breast cancer [1]. In particular, studies demonstrate that about one million women are diagnosed with the disease and that new cases are emerging at a rate of about 23\% [2]. To anticipate cases of cancer, some of the approaches that have received application include Helmholtz equation type and mathematical modeling [3, 4]. For the PDE under consideration, which has aided in disease anticipation in breast cancer, some of the models that have been used to solve it and give comprehensive insights include Alternating Group Explicit (AGE), Red Black Gauss Seidel (RBGS), and Jacobi (JB) methods [5, 6]. It is also notable that most of the parallel and sequential algorithms associated with numerical techniques have relied on MATLAB for their implementation, especially by using the distributed computing software [7, 8]. Also, the distributed parallel computer platform has been used to assist this software [6]. Indeed, the choice of the software has been determined by factors such as high speed processor performance and the huge memory allocation [9, 10]. To analyze the visualization of Helmoltz equation, numerical analyses that have been employed include the root 
mean square error (RMSE), maximum error, computational complexity, number of iteration, and the execution time [3-5]. In this study, focus was to analyze parallel performance evaluations relative to the variables of temporal performance, effectiveness, efficiency, speedup, and execution time. These analyses aided in paving the way for repository system development, in the form of a breast cancer repository.

\section{Methodology}

To detect tumor cell growth, this experimental investigation relied or focused on magnetic waves' biological aspects. With high performance computing (HPC) on the focus, the study sought to discern the feasibility of using Helmoltz equation in the early detection of breast cancer. This approach was taken because previous studies demonstrated that the technique exhibits precision for Helmoltz equation under infinity conditions, compared to scenarios where infinite physical domains involve the usage of Laplace's equations [3, 5]. The superiority of the model has been documented particularly to arise from the ability to ensure that the wave motion is presented. For the classification and detection of MRI cancer growth, the initial step involved the detection of the growth, followed by a second step in the form of pre-processing. To identify the type of cancer growth, methods that are used include feature selection and feature extraction.

\section{Results and Discussion}

As mentioned earlier, focus was on the performance of the parallel algorithm and the sequential algorithm in relation to their capacity for use in software development for the purpose of detecting breast cancer early enough. This chapter presents the results, with the initial section focusing on the outcomes that were obtained, following the implementation of the parallel algorithm and the sequential algorithm. When sequential algorithm was implemented, the flow of the calculations was meant to simulate breast cancer growth. In this algorithm, three major phases were involved and accomplished. Initially, there was the definition and initiation of the experiment's boundary and initial conditions. In the second phase, there was the validation of numerical solutions. This validation involved the use of a stopping criterion. In the third phase, there was the fulfillment of the stopping step in such a way that there was the implementation of the iterating procedure.

Regarding the case of the parallel algorithm, the setup was in such a way that slaves represented the processors while masters represented the servers, and there were communication activities among these attributes. For each processor, there was an assignment of sets of ranges. This step strived to ensure the computation of solutions. The ranges would then be sent via data structure decomposition processes. Indeed, it is worth indicating that through parallel computing, there emerges a privilege in such a way that multiple processors could execute the same task on a simultaneous basis. Given this simultaneous performance of roles by the processors, it also remains inferable that there tends to be a significant reduction in computational complexity's time execution. The figure below demonstrates the algorithm's functionality. 


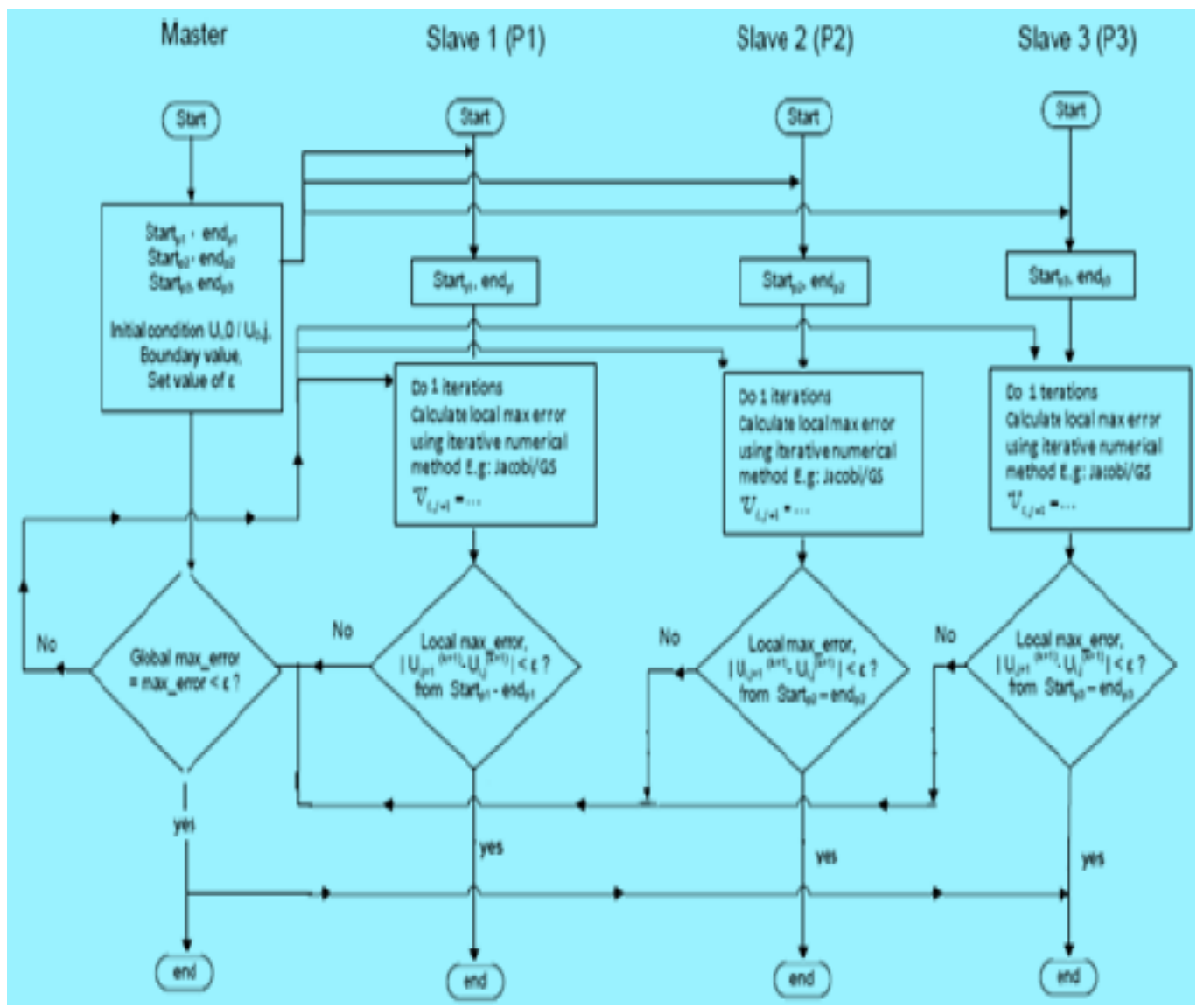

Before presenting the study's specific experimental outcomes that were obtained, it is also important to highlight the numerical analysis and the role it played in achieving the objective of the investigation. In this case, methods that were considered included Jacobi, RBGS, and AGE techniques. Specific parameters that were analyzed in relation to the incorporation of these numerical methods into the algorithms included RMSE, maximum error, computational complexity, number of iteration, and time execution. The following table provides a summary of the parameters that were measured, their values, and the interplay with the three selected numerical methods that were considered for incorporation into the functionality of the algorithms that were under investigation.

\section{Conclusion}

In summary, this study strived to demonstrate the criticality of using Helmoltz equation in relation to the analysis, classification, and prediction of the growth of breast cancer. For the numerical methods, AGE was found to exhibit superior outcomes, outperforming Jacobi and RBGS techniques. Therefore, AGE was affirmed to be an ideal numerical approach through which breast cancer growth could be handled or monitored. The AGE approach also outperformed the other two methods when additional parameters were evaluated. These parameters included maximum error, time execution, and number of iteration. Furthermore, AGE utilized half time step relative to the solution's computation, proving its superiority and feasibility. In situations where a large sparse matrix characterizes the given data at hand, parallel implementation outcomes are seen to be more promising than those produced by sequential algorithm. Hence, parallel algorithm could be used as an ideal model of solving sequential algorithm's drawbacks, especially regarding time consumption. 


\section{References}

[1] Mun, G.-J., B.-N. Noh and Y.-M. Kim, Enhanced stochastic learn-ing for feature selection in intrusion classification. International Journal of Innovative Computing, Information and Control, 2009. Five (11).

[2] Gladis Pushpa Rathi, V. and S. Palani, Brain tumor MRI image classification with feature selection and extraction using Linear Discriminant analysis. arXiv preprint arXiv:1208.2128, 2012.

[3] Rathi, V. and S. Palani, Brain tumor MRI image classification with feature selection and extraction using linear discriminant analy-sis. arXiv preprint arXiv:1208.2128, 2012.

[4] Tiwari, M.V. and D. Chaudhari, an Overview of Automatic Brain Tumor Detection from Magnetic Resonance Images. International Journal of Advanced Research in Engineering \& Technology (IJARET), 2013. 4(2): p. 61-68.

[5] Gunnarsson T. Microwave imaging of biological tissues: applied towards breast cancer tumor growth detection. Thesis 2007. De-partment of Compuhttps://doi.org/10.1016/j.fss.2008.11.016 ter Science and Electronics, Malaridalen University Vasteras

[6] Alias N, Ghani ACA., Saipansaipol HF., Ramli N, Palil SQM. Wave equation for early detection of breast cancer growth using MATLAB Distributed Computing. 2012 International Conference on Enabling Science and Nanotechnology, ESciNano 2012 - Pro-ceedings 2012.

[7] Alias, N., Alwesabi, Y., and Al-Rahmi, W. M. (2017). Chronology Of Brain Tumor Classification Of Intelligent Systems Based On Mathematical Modeling, Simulation And Image Processing Tech-niques.

[8] Eryılmaz, M., Önder . Ertan, F. . Yalçınkaya, and E. . Kara. "A Prediction Study about the Pandemic Era Based on Machine Learning Methods". International Journal on Recent and Innovation Trends in Computing and Communication, vol. 9, no. 12, Dec. 2021, pp. 01-07, doi:10.17762/ijritcc.v9i12.5492.

[9] Wang, Q., et al., Classification of brain tumors in MR imag-es. Pennsylvania State University, 2009.

[10] Khotanlou, H., et al., 3D brain tumor segmentation in MRI using fuzzy classification, symmetry analysis and spatially constrained deformable models. Fuzzy Sets and Systems, 2009. 160 (10): p. 1457-1473. https://doi.org/10.1016/j.fss.2008.11.016

[11] Yang, W. and M. Siliang. Automatic detection and segmentation of brain tumor using fuzzy classification and deformable models. In Biomedical Engineering and Informatics (BMEI), 2011 4th In-ternational Conference on. 2011. IEEE

[12] Al-Ansi, A. M. . (2021). Applying Information Technology-Based Knowledge Management (KM) Simulation in the Airline Industry . International Journal of New Practices in Management and Engineering, 10(02), 0509. https://doi.org/10.17762/ijnpme.v10i02.131 